\title{
Search strategies for charged Higgs bosons in CMS
}

\author{
Michele Gallinaro* ${ }^{* \dagger}$ \\ Laboratório de Instrumentação e Física Experimental de Partículas \\ LIP Lisbon \\ E-mail: michgallecern.ch
}

Strategies for the search of a charged Higgs boson with the CMS detector at the Large Hadron Collider are discussed. Whether it is "light" or "heavy", the charged Higgs boson can be discovered at the LHC, if it exists. With early data, the sensitivity to searches for a light Higgs boson is within reach. The large top quark production cross section at the LHC offers the opportunity to search for the charged Higgs boson as the $t \rightarrow H^{+} b \rightarrow \tau^{+} v v b$ decay can be large in extensions of the Standard Model, if the $H^{ \pm}$mass is lighter than the top quark mass, i.e. $m_{H^{ \pm}}<m_{\text {top }}$. A search for the charged Higgs boson in events with one lepton (electron or muon), at least two jets, large missing transverse energy, and one tau lepton can be performed for charged Higgs masses between 80 and $160 \mathrm{GeV} / \mathrm{c}^{2}$.

Third International Workshop on Prospects for Charged Higgs Discovery at Colliders - CHARGED2010, September 27-30, 2010

Uppsala Sweden

\footnotetext{
* Speaker.

${ }^{\dagger}$ on behalf of the CMS collaboration.
} 


\section{Introduction}

The Higgs boson may be at the origin of the mechanism through which particles acquire mass, but it has not been yet detected experimentally. Extensions to the standard model (SM) include a Higgs sector with two Higgs doublets, which include the charged $\left(H^{ \pm}\right)$as well as neutral $\left(h, H^{0}, A\right)$ Higgs bosons. If the charged Higgs is lighter than the top quark, i.e. $m_{H^{ \pm}}<\left(m_{\text {top }}-m_{b}\right)$, the MSSM and SM decay modes, i.e. $t \rightarrow H^{+} b$ and $t \rightarrow W^{+} b$, will compete. As a consequence, top quark pair production and decay would be a source of charged Higgs bosons through the channels $t \bar{t} \rightarrow W^{ \pm} H^{\mp} b \bar{b}$ and $t \bar{t} \rightarrow H^{+} H^{-} b \bar{b}$. This is the case of a "light" charged Higgs. Due to the large top production cross section at the LHC, this channel has a good discovery potential. The "heavy" charged Higgs, i.e. with a mass larger than the top quark mass, will not be easily accessible with early data. A study performed with simulated data at $\sqrt{s}=14 \mathrm{TeV}$ [1] indicates that at least approximately $30 \mathrm{fb}^{-1}$ are needed to signal the presence of a heavy charged Higgs boson in data, in the $g g \rightarrow t b H^{ \pm}$channel, with $H^{ \pm} \rightarrow \tau^{ \pm} v$ or $H^{ \pm} \rightarrow t b$. Due to the large amount of data needed, the sensitivity to this channel is not likely to be reached in the first few years of operation. Therefore, this search is not discussed further in this manuscript.

An absolute lower limit on the charged Higgs boson mass of about $80 \mathrm{GeV} / \mathrm{c}^{2}$ exists from LEP [2], independently of the branching ratios of the charged Higgs. Experimental results from the Tevatron collider at Fermilab have excluded the charged Higgs boson near the LEP mass limit of about $80 \mathrm{GeV}$ for small $(\leq 1)$ and large $\tan \beta$ values $(\geq 30)$ in the MSSM.

The search for the charged Higgs can be performed at the LHC. In particular, the study of $t \bar{t}$ events can play an important role, given the large $t \bar{t}$ production cross section. The large coupling of the charged Higgs to tau leptons can significantly alter the SM branching ratios, and could indicate the presence of the charged Higgs, or eventually help improving the current exclusion limits from the LEP and from the Tevatron experiments. In the following, a brief summary of the plans and strategies at the CMS experiment is outlined. With the current samples collected in 2010, the first data have been used as a preparatory ground to help understanding the detector and to perform the first measurements aiming at the "re-discovery" of the SM. Leptons, jets, and other kinematical quantities of the events, such as for example missing transverse energy, have been studied in detail and the corresponding SM measurements have been performed. All measurements performed so far are in good agreement with SM expectations. The preparation of the search for charged Higgs is based on the reliability of all reconstructed objects, as well as the full understanding of the techniques used. Tau identification and background determination using data-driven techniques have therefore been studied first. Later and with more data, indication on the presence of the charged Higgs can be addressed directly. At the time of writing, only preliminary studies are available and no deviations from the SM have been detected.

\section{The Large Hadron Collider and the experimental apparatus}

The Large Hadron Collider (LHC) at CERN has been colliding beams of protons at a center of mass energy of $7 \mathrm{TeV}$ in 2010 and delivered approximately $40 \mathrm{pb}^{-1}$ of data. The two main experiments, ATLAS and CMS, have been collecting data ever since with high efficiency, and the first physics results have been presented, rapidly showing a good understanding of the detector and 
of the main SM processes. These will become the sources of the backgrounds in the search for New Physics. During the next two years, 2011 and 2012, the LHC will continue collide proton beams and it will deliver a much larger data sample; with some optimism, it may be up to 100 times larger than what was delivered in 2010 alone.

\section{Search for charged Higgs in top quark events}

At the LHC, the large top quark production cross section offers a great opportunity to search for the charged Higgs boson. Top quarks are mostly produced in pairs. The measured production cross section in $p p$ collisions at $\sqrt{s}=7 \mathrm{TeV}$ is $\sigma_{t \bar{t}}=194 \pm 72$ (stat) \pm 24 (syst $) \pm 21$ (lum) pb [3]. At the nominal LHC energies of $\sqrt{s}=14 \mathrm{TeV}$, the top quark pair production cross section will be about 100 times larger than at the Tevatron [4].

In the SM, the top quarks decay to a bottom quark and a $W$ boson. The decay modes of the $W$ boson pair determine the observed event signature. The "standard dilepton" decay channel denotes the case where both $W$ bosons from the decaying top quark pair decay leptonically, i.e. to electrons or muons plus corresponding neutrinos, $t \bar{t} \rightarrow W^{+} W^{-} b \bar{b} \rightarrow\left(\ell v_{\ell}\right)\left(\ell v_{\ell}\right) b \bar{b}$, where $\ell=e, \mu$. With an analogously large branching ratio, the "tau dilepton" channel is sensitive to the presence of the charged Higgs. In this channel, one $W$ decays into $e v_{e}$ or $\mu v_{\mu}$, and the other into third-generation leptons $\tau \nu_{\tau}$. Consequently, the total decay chain is: $t \bar{t} \rightarrow W^{+} W^{-} b \bar{b} \rightarrow\left(\ell v_{\ell}\right)\left(\tau \nu_{\tau}\right) b \bar{b}$. The expected rate of the tau dilepton channel is approximately 6\% (5/81) of all $t \bar{t}$ decays, which is equivalent to the "standard" (ee, $\mu \mu, e \mu) t \bar{t}$ dilepton channel. A test of lepton universality in top quark decay final states may help setting limits on new non-SM physics processes. The tau dilepton channel is of particular interest because the existence of a charged Higgs with $m_{H^{ \pm}}<m_{t o p}$ could give rise to anomalous tau lepton production, which could be directly observable in this decay channel. At the Tevatron only a few events have been observed in the top dilepton channel with tau leptons in the final state [5,6]. Understanding tau production is therefore important for several reasons: a) to check universality of lepton couplings, b) to increase acceptance for $t \bar{t}$ events, and c) to search for new physics processes. In particular, this decay channel involves only leptons and quarks of the third generation, i.e. top and b-quarks, and tau leptons.

In some models, the different Higgs decay mechanisms are described in terms of the $\tan \beta$ parameter. For example, at large values of $\tan \beta$ (i.e. $\tan \beta>30) B R\left(H^{+} \rightarrow \tau v\right)=1$ (tauonic mode), whereas at small values (i.e. $\tan \beta \leq 1) B R\left(H^{+} \rightarrow c \bar{s}\right)=1$ (tauphobic mode). However, it is preferable to follow a model-independent approach, and parametrize the Higgs decays in terms of the branching ratio $B R\left(t \rightarrow H^{+} b\right)$, where the Higgs decay is assumed.

\section{Tau identification}

Tau leptons decay promptly either to lighter leptons or to hadronic jets, with corresponding branching ratios of $B R\left(\tau \rightarrow \ell v_{\ell} v_{\tau}\right) \approx 36 \%$ and $B R\left(\tau \rightarrow h \nu_{\tau}\right) \approx 64 \%$ (50\% for 1-prong, and $14 \%$ for 3-prong decays). The identification of hadronic tau decays is difficult due to the background contributed by the much more abundant quark or gluon jets. This is especially true at hadron colliders, where the hadronic activity in the final states is large. The case in which the taus decay leptonically is experimentally difficult to distinguish from prompt electron or muon production, 
except that the spectrum of the lepton transverse momentum is softer in the case of sequential decays.

Several algorithms for reconstruction and identification of hadronic tau decays are used within CMS [7]. Tau candidates are reconstructed using tracking and calorimeter information. The algorithms use simple discriminating variables to distinguish between genuine hadronic tau decays and generic QCD jets by subdividing jets into two cones: a narrow signal cone, expected to include all tau decay products, and an isolation cone surrounding the signal cone, where no particles above a certain threshold are present. The Track-Corrected Tau (TCTau) algorithm is based on jets reconstructed from tracks and energy deposits in the electromagnetic and hadronic calorimeters, with a signal cone of fixed size $\Delta R=0.07$. The "shrinking cone" algorithm is based on particle flow (PF) jets with a variable signal cone size $\left(\Delta R=5.0 / E_{T}\right)$, where the angle between tau decay products is adjusted according to the Lorentz boost expected for different tau momenta, thus improving the identification efficiency for tau hadronic 3-prong decays. More complex algorithms aim at reducing the rate by which generic QCD jets get mis-reconstructed as tau candidates by analyzing the constituents of jets in order to identify specific tau hadronic decay modes, by taking full advantage of the performance provided by PF techniques for reconstructing individual charged hadrons and photons. The Hadron plus Strips (HPS) algorithm employs cuts on the multiplicity and invariant mass of charged hadrons and neutral pions reconstructed in a narrow cone, together with the requirement that there be no other particles of $p_{T}$ above a certain threshold within the jet. The neutral pions are reconstructed using topological criteria which account for photon conversion effects. The Tau Neural Classifier (TaNC) algorithm reconstructs neutral pions by combining photon pairs of low invariant mass. The discrimination between tau lepton hadronic decays and generic QCD jets is based on an ensemble of five neural networks which have been trained to identify a particular hadronic decay mode each. In order to satisfy the requirements of different physics analyses on tau identification efficiency versus fake rate, a set of different "working points" is provided.

The performance of the different algorithms is compared in terms of "fake rate" and efficiency. The fake rates of the TCTau, shrinking cone, HPS and TaNC tau identification algorithm are measured as a function of jet $p_{T}$ and $\eta$ by counting the fraction of quark and gluon jets passing the identification criteria of one particular algorithm in a given $p_{T}$ jet or $\eta$ bin. The fake rates measured for the four tau identification algorithms are presented in Fig. 1 (left). The efficiencies to identify genuine hadronic tau decays, estimated using a sample of simulated $Z \rightarrow \tau \tau$ events are shown in Fig. 1 (right).

\section{Tauphobic decay mode}

At low $\tan \beta$, in the case where the charged Higgs decays through $H^{+} \rightarrow c \bar{s}$, the final state of the decay $t \bar{t} \rightarrow W^{ \pm} b H^{\mp} b$ will include one high- $p_{T}$ isolated lepton (electron or muon), missing transverse energy and at least 4 jets, of which two are from the hadronization of $b$-quarks. This final state is similar to the SM semi-leptonic $t \bar{t}$ decay, where only one $W$ decays leptonically while the other decays hadronically. A direct search for a dijet mass resonance is difficult. Instead, the effect that the decay mode $t \rightarrow H b$ would have on $t \bar{t}$ decay rates to eev $v+$ jets, $\mu \mu \nu v+$ jets, $e \mu \nu v+$ jets (i.e. "dilepton") final states, as well as $e v+$ jets and $\mu \nu+$ jets (i.e. "lepton+jets") final states can be studied. The comparison of the observed number of events in data with the 

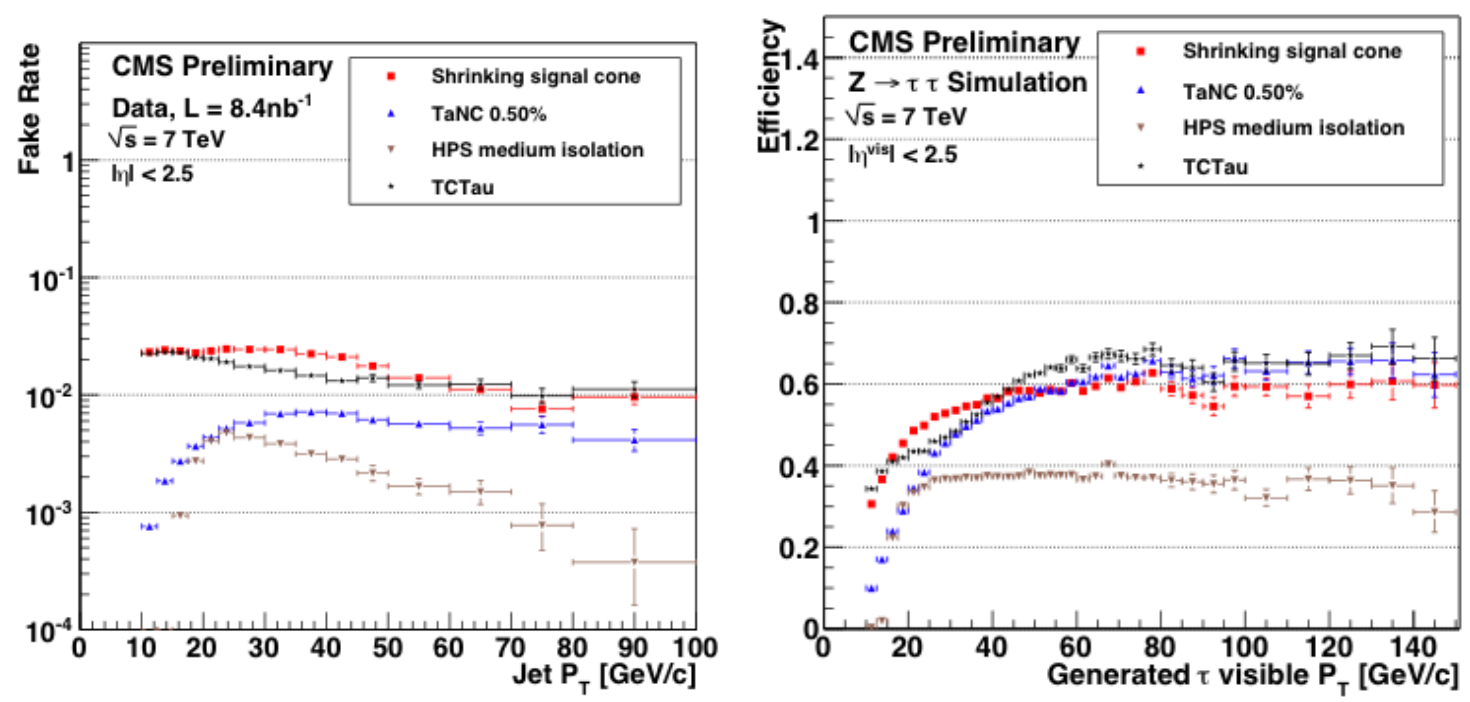

Figure 1: Left: Measured probabilities of quark/gluon jets to pass the tau candidate selection criteria of the TCTau (black stars), shrinking cone (red squares), HPS (brown downward facing triangles) and TaNC (blue upward facing triangles) tau identification algorithms as a function of jet $p_{T}$, estimated from a QCD multi-jet data sample. Right: Efficiencies of the algorithms to identify genuine tau lepton hadronic decays as a function of the transverse momentum of the visible tau decay products at the generator level, using a sample of simulated $Z \rightarrow \tau \tau$ events.

expected number of events, assuming that $t \bar{t}$ pairs decay exclusively to $W b W b$ provides indication of the presence or absence of the charged Higgs. Furthermore, by including different and exclusive event categories, it is possible to improve the sensitivity to the charged Higgs [8].

\section{Reconstruction and event selection}

The top quark sample is an important starting sample to perform the study for the search for light charged Higgs. Electrons, muons, jets and taus are reconstructed using the standard algorithms already used in the early analyses. The minimal inclusive lepton trigger used in the data taking run of 2010 provides with an almost un-biased starting sample. In 2010, the inclusive lepton data set was collected with triggers which require events to have at least one electron or muon above a minimum transverse momentum $\left(p_{T}\right)$ threshold. Due to the rapid increase of the instantaneous luminosity in 2010, the lepton $p_{T}$ thresholds have been increased during the data taking period in order to keep the trigger rates within the data acquisition capabilities. Most of the data were collected with thresholds of $p_{T}>15 \mathrm{GeV} / \mathrm{c}$ for muons and $p_{T}>17 \mathrm{GeV} / \mathrm{c}$ for electrons. In the years to come and for example in 2011-2012, the instantaneous luminosity is expected to rise from $L=10^{32} \mathrm{~cm}^{-2} \mathrm{sec}^{-1}$ up to $L=5 \cdot 10^{33} \mathrm{~cm}^{-2} \mathrm{sec}^{-1}$; under these conditions it will be difficult to maintain such a trigger without introducing an artificial reduction ("pre-scale") in the rate of the data collected. A trigger based on specific event topologies, or by selecting a combination of the objects in the given final state could be used, such as, for example, a "lepton+tau" trigger with appropriate thresholds for leptons and taus. 
Muons are reconstructed [9] using a simultaneous global fit performed to hits in the silicon tracker and the muon system. Electron candidates are reconstructed [10] starting from a cluster of energy deposits in the crystals of the ECAL which is then matched to hits in the silicon tracker. Leptons are required to be isolated from other activity in the event. Jets and missing transverse momentum are reconstructed using the PF algorithms [11]. Additional details on the reconstruction algorithms used in the early data can be found in [3].

Tau dilepton events are characterized by the presence in the final state of two leptons (one electron or muon, and one tau), two jets from $b$-quark hadronization, and missing transverse energy $\mathscr{E}_{T}$ from the neutrinos in the event. Events are selected by requiring at least one high- $p_{T}\left(p_{T}>\right.$ $20 \mathrm{GeV} / \mathrm{c}$ and $|\eta|<2.4)$ and isolated lepton $\left(e\right.$ or $\mu$ ), at least two jets with $p_{T}>30 \mathrm{GeV} / \mathrm{c}$ and $|\eta|<2.4$, large missing transverse energy $\left(E_{T}>40 \mathrm{GeV}\right)$ from the neutrinos, and one tau lepton $\left(p_{T}^{\tau-j e t}>20 \mathrm{GeV} / \mathrm{c}\right.$ and $|\eta|<2.4$ ). All objects are required to be separated in the $\eta-\phi$ space by $\Delta R>0.3$. The inclusive lepton is required to be isolated both in the calorimeter and in the tracker. The calorimeter isolation takes into account the energy deposited in a cone of $\Delta R=0.3$ around the direction of the lepton. Tracking isolation is calculated by summing the transverse momenta of all tracks in a cone of radius $\mathrm{R}=0.3$ around the lepton's direction. Because of the large top quark mass, $t \bar{t}$ events exhibit large total transverse energy. The two high- $p_{T}$ isolated leptons in the $t \bar{t}$ dilepton decay come from the two $W$ bosons and are required to be oppositely charged.
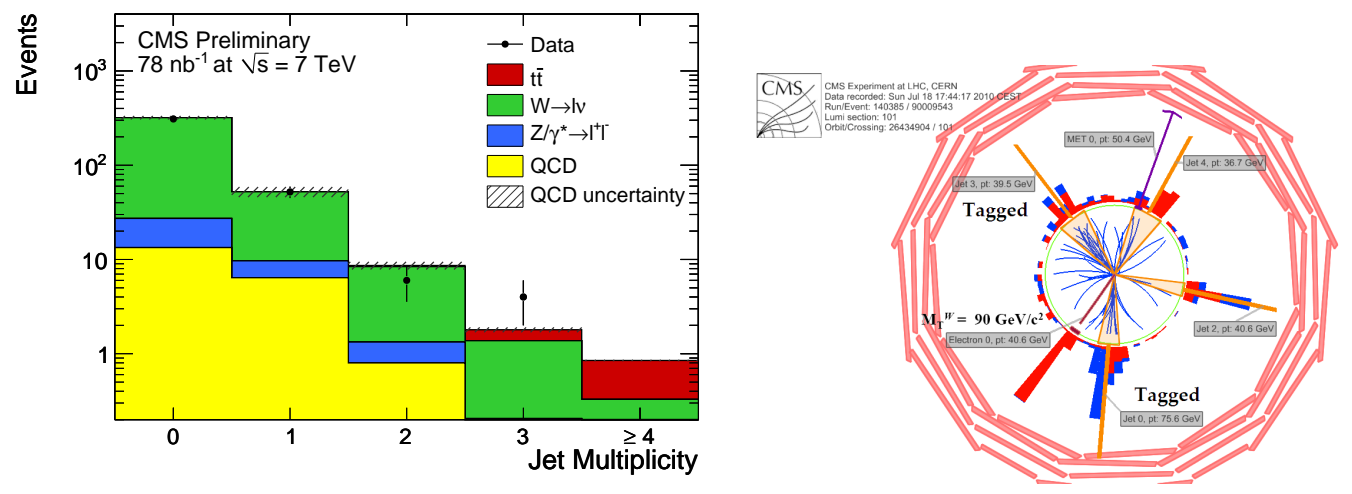

Figure 2: Left: Jet multiplicity distribution of events with one reconstructed muon (" $\mu+$ jet" channel), corresponding to $78 \mathrm{nb}^{-1}$ of data. Right: event display of one "electron+4 jets" candidate with two b-tagged jets, and one narrow jet ( $p_{T}=73 \mathrm{GeV} / \mathrm{c}, \eta=-1.3, \phi=-0.2$ ) with low track multiplicity (one high $p_{T}$ track is pointing at the jet), a characteristics of a tau hadronic decay.

\section{Backgrounds}

In the case of hadronic tau decays, the dominant source of the background comes from "tau fakes", i.e. where one jet is misidentified as a tau. The data-driven method used in the estimation of the tau-dilepton fake events is described in [12]. This background comes mainly from two sources: a) events where one $W$-boson is produced in association with jets, and b) $t \bar{t}$ events, where the $W$ and the jets are mostly from top decays. In order to estimate the contribution of this background 
directly from data, a two-step process is used. First, the probability that a jet "fakes" a hadronically decaying tau lepton is evaluated from all jets in a jet-dominated sample (i.e. multi-jet or photon+jet), and is parametrized as a function of jet $p_{T}$. The "fake" rate is calculated from the ratio of the $p_{T}$ distribution of the jets which have satisfied the tau-tagging algorithm divided by the inclusive jet $p_{T}$ distribution. This ratio gives the probability that each jet fakes a hadronically decaying tau lepton. Finally, the number of fake events is obtained by applying the fake probability to the $p_{T}$ spectrum of all jets in the events passing the $W+\geq 3$ jet selection. Preliminary results indicate that a 15\%-20\% agreement with the expected "tau fakes" background can be found.

In the case of leptonic tau decays, the main sources of background come from $t \bar{t}$ and Drell-Yan events. Studies of these backgrounds may also be estimated from data [3].

\section{Prospects and conclusions}

The first year of operations of proton-proton collisions at $\sqrt{s}=7 \mathrm{TeV}$ at the LHC in 2010 has been outstanding in terms of detector and machine performance. In the next few years of operations, it is expected that much larger data samples will be collected by the experiments.

The first physics measurements have already been obtained and presented by both ATLAS and CMS experiments, and good agreement is found with SM expectations. The light charged Higgs, i.e. $m_{H^{ \pm}}<m_{t o p}$, may be one of the first searches which can already be probed with early data. The $t \bar{t}$ sample and the decay channels including tau leptons, especially hadronic tau decays, will play a crucial role in the search for the charged Higgs. Results obtained with the early data indicate that the tau efficiencies and fake rates are understood, and the techniques for charged Higgs searches are been refined. Lessons from the previous experiments will certainly play an important role.

\section{Acknowledgments}

Thanks to my colleagues in the CMS experiment for the indefatigable efforts towards an early advanced understanding of the detector performance and preparation of the first physics results, and to all involved in the LHC accelerator complex for an outstanding performance of the machine. Thanks to the organizers of the workshop for a nice atmosphere and an stimulating workshop in a secluded and quiet place.

\section{References}

[1] G. L. Bayatian et al. [CMS Collaboration], "CMS technical design report, volume II: Physics performance," J. Phys. G 34 (2007) 995.

[2] ALEPH, DELPHI, L3 and OPAL Collaborations, The LEP working group for Higgs boson searches, "Searches for Higgs bosons: Preliminary combined results using LEP data collected at energies up to $202 \mathrm{GeV}$," CERN-EP/2000-055.

[3] V. Khachatryan et al. [CMS Collaboration], "First measurement of the cross section for top quark pair production in proton-proton collisions at $\sqrt{s}=7 \mathrm{TeV}$," arXiv:1010.5994 [hep-ex].

[4] M. Cacciari, S. Frixione, M. Mangano, P. Nason, G. Ridolfi, "Updated predictions for the total production cross sections of top and of heavier quark pairs at the Tevatron and at the LHC," JHEP 0809 (2008) 127. 
[5] A. A. Affolder et al. [CDF Collaboration], "Search for the charged Higgs boson in the decays of top quark pairs in the $e \tau$ and $\mu \tau$ channels at $\sqrt{s}=1.8$ TeV," Phys. Rev. D 62 (2000) 012004 [arXiv:hep-ex/9912013].

[6] A. Abulencia et al. [CDF Collaboration], "A search for $t \rightarrow \tau v q$ in $t \bar{t}$ production," Phys. Lett. B639 (2006) 172.

[7] CMS collaboration, "Study of tau reconstruction algorithms using $p p$ collision data collected at $\sqrt{s}=7$ TeV," CMS-PAS-PFT-10-004.

[8] V. M. Abazov et al. [D0 Collaboration], "Search for charged Higgs bosons in top quark decays," Phys. Lett. B 682, 278 (2009) [arXiv:0908.1811 [hep-ex]].

[9] CMS collaboration, "Performance of muon identification in $p p$ collisions at $\sqrt{s}=7 \mathrm{TeV}, "$ CMS-PAS-MUO-10-002",

[10] CMS collaboration, "Electron reconstruction and identification at $\sqrt{s}=7 \mathrm{TeV}$," CMS-PAS-EGM-10-004.

[11] CMS collaboration, "Commissioning of the particle flow reconstruction in minimum-bias and jet events from $p p$ collisions at $7 \mathrm{TeV}$," CMS-PAS-PFT-10-002.

[12] CMS collaboration, "Towards the measurement of the $t \bar{t}$ cross section in the $e \tau$ and $\mu \tau$ dilepton channel in $p p$ collisions at $\sqrt{s}=14$ TeV," CMS-PAS-TOP-08-004. 